\title{
Rendimiento académico en función del grado de exigencia
}

\author{
Ramón Sarrias-Ramis, Jorge Pérez
}

Objetivo. Determinar si un nivel de exigencia superior para pasar una asignatura aumenta el rendimiento de los estudiantes.

Sujetos y métodos. En los estudios de medicina de la Universitat Autònoma de Barcelona, se compararon los resultados en dos asignaturas amplias de segundo curso con las mismas características, pero con grado de exigencia diferente para superar la asignatura.

Resultados. Los resultados fueron muy similares en ambas situaciones. No aparecieron diferencias en el rendimiento de los alumnos en las dos asignaturas.

Conclusiones. Una superior exigencia no implicó aumento de rendimiento. Posiblemente se debería a que los estudiantes de medicina, motivados y brillantes, generalmente se esfuerzan al máximo.

Palabras clave. Estándar para superar una asignatura. Estudiantes de medicina. Evaluación.

\section{Requirement to pass a subject and academic achievement}

Aim. To determine if a higher requirement to pass a subject increases student learning.

Subjects and methods. With medical students at Universitat Autònoma de Barcelona, we compared the results of two large second-year subjects with the same features but with different degree of requirement to pass the subject.

Results. The results were very similar in both situations. No differences in student performance were found in the two subjects.

Conclusions. A higher requirement did not increase the student achievement. Possibly would be because medical students, motivated and bright, usually try to achieve their best.

Key words. Assessment. Medical students. Pass-fail standards.

\section{Introducción}

Un tema importante en la docencia universitaria es el establecimiento de los estándares mínimos para superar una materia. Sabemos que existen dos posibilidades para fijar los criterios de superación de una asignatura. Con los internos o relativos, un alumno determinado supera o no la asignatura en función del rendimiento del grupo. A partir de la distribución de las puntuaciones (campana de Gauss) se marca el porcentaje de estudiantes que superarían una determinada prueba. Por el contrario, el criterio externo o absoluto se basa en criterios marcados a priori $[1,2]$. Un estudiante aprobará la materia siempre que supere los criterios marcados. Este es el modelo más utilizado y el más adecuado desde el punto de vista educativo [1].

Considerando la opción del criterio externo, el debate se traslada a cuáles han de ser los aprendizajes mínimos, traducidos en puntuaciones mínimas en los exámenes, para superar los procesos docentes [3].
Sobre este tema hay posturas que consideran que cuanto más elevados sean los estándares, mayor será el aprendizaje de los estudiantes. Contrariamente, otros profesionales consideran que unos estándares muy elevados aumentarían el fracaso académico sin mejorar los aprendizajes, especialmente con estudiantes brillantes y motivados.

Este tema es muy difícil de estudiar empíricamente por los problemas éticos que se podrían derivar a la hora de modificar o de ocultar información al estudiante en relación a los criterios mínimos de exigencia para superar una asignatura.

Una circunstancia coyuntural en los estudios de medicina de la Universitat Autònoma de Barcelona (UAB) permitió la realización de este estudio. En una determinada asignatura, por error, se comunicó a los estudiantes una exigencia más elevada a la prevista. Dicho error se detectó una vez realizado el examen, pero antes de su corrección. Este hecho permitió conocer el resultado de los exámenes de dos asignaturas prácticamente idénticas donde, $a$
Facultad de Ciencias de la Salud y de la Vida. Universitat Pompeu Fabra. Barcelona, España.

Correspondencia:

Dr. Jorge Pérez Sánchez. Facultad de Ciencias de la Salud y de la Vida. Universitat Pompeu Fabra. Doctor Aiguader, 80. E-08003 Barcelona.

E-mail:

jordi.perez@upf.edu

Conflicto de intereses: No declarado.

Competing interests: None declared.

(c) 2015 FEM 
priori, se había informado a los estudiantes un nivel de exigencia diferente.

El objetivo de este estudio fue observar posibles diferencias en el rendimiento de los alumnos ante la presencia de dos niveles de exigencia distintos en dos asignaturas de características similares.

Dado el tipo de estudiantes donde se realizó el estudio, brillantes y motivados por la carrera, se efectuó la hipótesis de que el rendimiento en ambas situaciones sería similar.

\section{Sujetos y métodos}

\section{Ubicación de la experiencia}

La experiencia objeto de este estudio se realizó en la Facultad de Medicina de la UAB durante el curso 1995-1996. En aquel momento, los estudios de medicina tenían 511 créditos y se cursaban en 12 semestres. El currículo contemplaba asignaturas amplias con contenidos de diferentes materias [4]. En el segundo curso de carrera existían dos asignaturas denominadas 'Desarrollo, estructura y función de los aparatos' y 'Sistemas en estado de salud'. La primera (AS-1) tenía 33 créditos y se cursaba en el primer trimestre; la segunda (AS-2), 36 créditos y se cursaba en el segundo trimestre.

Ambas asignaturas tenían contenidos de anatomía, histología y fisiología y eran impartidas por los mismos profesores con características docentes prácticamente idénticas.

En la primera asignatura se había comunicado por error que, para aprobarla, los estudiantes deberían obtener una nota de 6. Después de los exámenes, pero antes de la corrección, se detectó el error, ya que el estándar adoptado por la facultad era de 5. Las evaluaciones se realizaron con el estándar previsto de 5. En la segunda asignatura, los estudiantes fueron informados de forma correcta desde el principio.

\section{Participantes}

Los resultados del estudio provenían de los estudiantes matriculados en las asignaturas AS-1 y AS-2 de segundo curso de medicina en la UAB durante el curso académico 1995-1996.

\section{Material}

Para la obtención de los datos, se utilizaron los resultados informatizados de las pruebas de elección múltiple (PEM) de ambas asignaturas en su primera convocatoria.

\section{Procedimiento}

Se compararon los resultados en las PEM entre ambas asignaturas. Para el estudio sólo se consideraron los resultados sin incidencias técnicas. Las hojas de respuestas con problemas de lectura se corrigieron a mano y no se consideraron para el estudio.

La diferencia de criterio consistía en que en AS-1 los estudiantes pensaban que el estándar (un 6) era superior que el previsto en AS-2 (un 5).

Para comparar los resultados se utilizó la prueba de chi al cuadrado mediante los criterios de evaluación que eran habituales en la Facultad de Medicina de la UAB (suspenso < 5; aprobado: 5-6,9; notable: 7-7,9; sobresaliente: $\geq 8$ ).

\section{Resultados}

En la tabla se puede observar el número de alumnos y el porcentaje en las diversas categorías. El porcentaje en cada apartado de notas fue muy similar y no existieron diferencias significativas entre ambas situaciones $\left(\chi^{2}=2,19\right.$; g.l. $\left.=3 ; p=0,53\right)$.

Utilizando sólo un criterio dicotómico, éxito (nota $\geq 5$ ) y fracaso (nota $<5$ ), tampoco existieron diferencias: $71,8 \%$ y $28,2 \%$ en AS- 1 frente a $72,4 \%$ y $27,6 \%$ en AS- 2 , respectivamente $\left(\chi^{2}=0,02\right.$; g.l. $=1$; $p=0,87)$.

A pesar de que la exigencia era diferente a la hora de realizar la PEM, el rendimiento fue muy similar.

\section{Discusión}

En primer lugar debe comentarse la dificultad de realizar un estudio como éste por los problemas éticos que pudieran derivarse de realizar cambios en las normas de evaluación de los estudiantes. Por suerte, la situación azarosa y excepcional que se ha comentado, y que en ningún caso perjudicó a los alumnos, permitió disponer de la información presentada.

Las dos asignaturas objeto de comparación tenían las mismas características referentes al tipo y número de profesores, contenidos de aprendizaje y metodologías docentes. Así, la comparación realizada es pertinente para el objetivo, ya que la única diferencia entre ambas situaciones consistió en la información dispensada a los estudiantes sobre el estándar para superar la asignatura.

A pesar de que en las dos asignaturas estudiadas había más elementos de evaluación que los de la PEM, el trabajo se ha realizado exclusivamente con 
los resultados en dicha prueba, ya que es totalmente objetiva y no se presta a posibles distorsiones debidas al evaluador [1-3,5].

Los resultados son claros y contundentes al indicar que, en el caso estudiado, un nivel superior de exigencia no se tradujo en un superior rendimiento de los alumnos. Dichos resultados fueron acordes con nuestras previsiones porque creemos que los estudiantes muy motivados y trabajadores, como es en general el caso de los estudios de medicina, tienden a hacer el máximo esfuerzo para obtener los mejores resultados.

El trabajo presentado tiene algunas limitaciones. Se puede observar que el número de estudiantes en la prueba de AS-2 es inferior al de AS-1. Ello se explicaría por un superior número de incidencias de lectura óptica en la corrección de la segunda prueba. Asumimos que dichos problemas se distribuirían al azar respecto al rendimiento de la prueba. De hecho, comprobamos que en las actas académicas definitivas, donde se consideraban todos los criterios de evaluación, no existieron diferencias en las calificaciones de ambas asignaturas.

Creemos que una limitación importante de nuestro estudio es el tiempo transcurrido desde que se realizó hasta nuestros días, ya que podría ponerse en duda si las características de los estudiantes de entonces y de ahora serían similares. Nuestra experiencia se inclina por considerar que los estudiantes de medicina actuales mantienen las mismas peculiaridades que los de hace unos años, especialmente en capacidad y motivación. Recordamos que en los momentos del estudio ya había una fuerte demanda para estudiar medicina y, por tanto, una gran selectividad. Además, hemos considerado que los resultados del estudio sobre un tema importante y producto de una situación azarosa podrían ser de utilidad y, por tanto, deberían ser conocidos por la comunidad académica.

Queremos hacer notar que una exigencia excesiva es una de las causas más importantes del fracaso académico, si bien es percibida de forma diferente por los profesores que por los alumnos. Los prime-
Tabla. Número de estudiantes y porcentaje en las diversas categorías de rendimiento en las dos asignaturas.

\begin{tabular}{lcc}
\hline & AS-1 $(n=266)$ & AS-2 $(n=225)$ \\
\hline Suspenso & $75(28,2 \%)$ & $62(27,6 \%)$ \\
\hline Aprobado & $133(50,0 \%)$ & $103(45,8 \%)$ \\
\hline Sotable & $38(14,3 \%)$ & $43(19,1 \%)$ \\
\hline Sobresaliente & $20(7,5 \%)$ & $17(7,5 \%)$
\end{tabular}

AS-1: asignatura con requisito de 6 para aprobar; AS-2: asignatura con requisito de 5 para aprobar.

ros conceden menos importancia a dicha exigencia que los estudiantes [6].

En conclusión, consideramos que sin renunciar a la gran exigencia que requieren los estudios de medicina no se ha de poner el acento en incrementarla de forma irreflexiva porque posiblemente no aumentaría el aprendizaje, pero sí el fracaso académico. Sería más adecuado buscar los estándares mínimos adecuados a partir de objetivos docentes.

\section{Bibliografía}

1. Wass V, Archer J. Assessing learners. In Dornan T, Mann K, Scherpbier A, Spencer J, eds. Medical education. Theory and practice. Edinburgh: Churchill Livingstone/Elsevier; 2011. p. 229-55.

2. Guilbert JJ. Guía pedagógica para el personal de salud. 6 ed. Valladolid: Organización Mundial de la Salud-Universidad de Valladolid; 1994.

3. Epstein RM. Assessment in medical education. N Engl J Med 2007; 356: 387-96.

4. Pérez J, Sarrias-Ramis R, Reig J. Evaluación del rendimiento académico en asignaturas con contenidos amplios: global vs. por áreas de conocimiento. Educ Med 1998; 1: 63-7.

5. Palés-Argullós J. ¿Cómo elaborar correctamente preguntas de elección múltiple? Educ Med 2010; 13: 149-55.

6. Tejedor FI, García-Valcárcel A. Causas del bajo rendimiento del estudiante universitario (en opinión de los profesores y alumnos). Propuestas de mejora en el marco del EEES. Rev Educ 2007; 342: 443-73. 